Artículo original

\title{
Etnografía del productor araucano de la sabana inundable, Colombia
}

\author{
$\odot$ Luis Ernesto Rodríguez-Qüenza ${ }^{1}$, Alonso Correa-Toro', $\odot$ Milena Hernández-Rodríguez ${ }^{2}$, ๑ Arcesio Salamanca C.,** \\ ${ }^{1}$ Facultad de Medicina Veterinaria y Zootecnia, Universidad Nacional de Colombia, sede Bogotá, Bogotá, D.C., Colombia \\ ${ }^{2}$ Grupo de Investigación Los Araucos, Facultad de Medicina Veterinaria y Zootecnia, \\ Universidad Cooperativa de Colombia, Arauca, Colombia
}

\section{Resumen}

El objetivo del presente artículo fue describir la etnografía del llanero habitante de los municipios de Arauca, Cravo Norte y Puerto Rondón, localizados en las sabanas inundables del departamento de Arauca, Colombia, a partir del enfoque sistémico de los diversos agroecosistemas presentes en ellos, de las formas de vida y la relación con la naturaleza, recurriendo a los conceptos de subjetividad e intersubjetividad de la investigación cualitativa, en un "diálogo de saberes" respaldado por entrevistas semiestructuradas con final abierto hechas a 30 productores, diez por municipio. Se seleccionaron 11 temas que ocupan gran parte de la cotidianidad llanera y que posibilitan la existencia de estos productores, para escucharlos de viva voz con la esperanza de construir propuestas concretas surgidas del sentir "llanero anfibio". Los entrevistados se clasificaron según el tamaño de sus predios y del inventario ganadero así: grandes productores (más de 1.000 bovinos), productores medianos (500 a 1.000 bovinos) y pequeños productores (menos de 500 bovinos). La información obtenida se interpretó desde las perspectivas subjetiva e intersubjetiva y a partir de allí se construyeron los resultados. El $70 \%$ de los entrevistados eran herederos directos de sus predios, muchos de ellos, fundadores, y solo el $30 \%$ del área total era "banqueada" (nivelada), el resto, baja. Predominaba el ganado cebuíno, pero las razas y las sabanas nativas eran también visibles; el 53,3\% de los productores ordeñaban y producían queso artesanal o vendían leche. La economía natural de las explotaciones tradicionales de Arauca y Cravo contrastó con las transformaciones sufridas por los agroecosistemas de los predios de Rondón, más cercanos al dinámico piedemonte. (C) 2019. Acad. Colomb. Cienc. Ex. Fis. Nat.

Palabras clave: Sabanas inundables; Sistemas; Conucos; Llaneros; Recursos nativos.

Ethnography of the Araucanian producer of the flooded savannah, Colombia

\begin{abstract}
The objective of this article was to describe the ethnography of the inhabitants of Arauca, Cravo Norte, and Puerto Rondón municipalities, located in the flooded savannahs of the Department of Arauca, Colombia, from a systemic approach of the different ecosystems present in them, the prevalent ways of life and the relationship with nature using qualitative research subjectivity and intersubjectivity criteria and open-end semi-structured interviews through a "dialogue of knowledges" with 30 producers, ten per municipality. We selected 11 subjects that are a central part of the "llanero" everyday life and allow for the existence of these producers in an attempt to listen to their voices and in the hope to build concrete proposals arising from the feelings of this "amphibious llanero". The interviewees were categorized according to the size of their land and their livestock inventory as follows: big producers (more than 1,000 heads of cattle), medium producers (500 to 1,000 heads of cattle), and small producers (less than 500 heads of cattle). We analyzed the information obtained using subjectivity and intersubjectivity criteria and from there, we established the results: $70 \%$ of the interviewees were direct heirs of their lands, many of them, founders. Only $30 \%$ of the total area corresponded to leveled land and the rest to low lands. The predominates cattle breed is zebu, but the native creole breeds are now more visible; $53.3 \%$ of the interviewees milked cows and produced artisanal cheese. The natural economy of the traditional farms in Arauca and Cravo Norte contrasted with the transformations occurred in the agro-ecosystems of Puerto Rondón municipality, which is closer to the dynamic piedmont areas. (C) 2019. Acad. Colomb. Cienc. Ex. Fis. Nat.
\end{abstract}

Key words: Seasonally flooded savannahs; Systems; Conucos; Llaneros; Native resources.

\footnotetext{
*Correspondencia:

Arcesio Salamanca Carreño, asaca_65@yahoo.es; Recibido: 30 de agosto de 2018; Aceptado: 27 de marzo de 2019; Editor: Rubén Ardila
} 


\section{Introducción}

El enfoque integral de sistemas de producción contribuye a los fines conservacionistas de salvaguarda del medio ambiente, pues las actividades productivas, por ejemplo la ganadería, en ecosistemas frágiles como los de las sabanas inundables pueden tener impactos insospechados. El enfoque integral es un método de reflexión y análisis que requiere de una observación analítica para conocer la forma en que se desarrollan dichas actividades productivas, así como las particularidades de las explotaciones tradicionales que, como la ganadería de cría, han estado estrechamente vinculadas al uso de la oferta natural, a la comprensión de la dinámica de los ciclos hidrológicos y a la movilidad de los animales en el territorio. Asimismo, este enfoque exige una aproximación integral y la comprensión de los factores determinantes de la producción, incluidos los aspectos culturales y sociales, aspectos que arrojan luz sobre la sostenibilidad de la actividad mediante el análisis de su impacto en la transformación de los territorios por el probable cambio en los usos del suelo. En este sentido, se recurrió a la revisión del enfoque sistémico y de sus aportes para entender mejor la realidad actual de la ganadería araucana y de las oportunidades que brinda para lograr un desarrollo más sustentable que conserve la funcionalidad ecosistémica de este paisaje orinoquense (Peñuela, et al., 2014).

Por otra parte, la teoría general de sistemas se ha convertido en una herramienta útil al propiciar la construcción de modelos útiles en diferentes campos y para evitar el uso de vagas analogías que han afectado el avance en los campos del conocimiento pertinentes. El sistema, o modelo, constituye una herramienta de análisis cuyo valor se aprecia incluso en casos en que no puede alcanzarse una formulación matemática, pues sigue siendo una "idea guía" que orienta el análisis. En el campo de la sociología, por ejemplo, la simple consideración de que las comunidades y organizaciones sociales son sistemas, no sumas de individuos, que se denominan civilizaciones y obedecen a los principios generales de los sistemas, implica una reorientación en sus estudios. Todo organismo vivo es, ante todo, un sistema abierto que incorpora y elimina continuamente materia, constituyendo y demoliendo componentes sin alcanzar, mientras dura la vida, un estado de equilibrio químico y termodinámico que se mantenga uniforme. La esencia del fenómeno fundamental de la vida, el metabolismo, está constituida por los procesos químicos que se verifican dentro de las células vivas. En el mundo real los sistemas son abiertos, es decir, tienen interacción con el ambiente (Hart, 1985). En el estudio de la producción agropecuaria, el enfoque de sistemas permite la integración de diversas disciplinas que, en el análisis de una situación productiva en particular, considere los aspectos biofísicos y socioculturales que configuran el escenario real donde operan los sistemas de producción agrícola (Malagón \& Prager, 2001).
Desde la perspectiva de la teoría general de sistemas, se entiende la finca ganadera como un proceso de transformación de energía solar que abarca desde el pasto hasta la carne, la lana o la leche para el consumo humano (Aguilera, 1989; Machado \& Campos, 2008; Ospina, 2016), y en el marco del imaginario multidimensional, en la naturaleza todo está interconectado tanto socioeconómica como socioculturalmente. (Jara, 2009). El enfoque integral conecta diferentes elementos, atendiendo a las jerarquías y enfocándose en los procesos (Ospina, 2016). La razón de adoptar esta herramienta es muy simple: hay poca esperanza de entender los procesos agrícolas si se siguen estudiando solamente los detalles. Muchos cultivos se utilizan para la alimentación humana y también para la animal. El sistema tiene una corriente de entrada que importa energía e insumos desde el entorno, los cuales se someten a un proceso de conversión en el que, por el efecto de la sinergia y la recursividad, se transforman y salen como insumos o productos exportados al entorno (Hart, 1985; Johansen, 2004). Por último, desde la perspectiva sistémica, la ganadería en la sabana inundable no puede ser un proceso aislado $\mathrm{y}$, como tal, sus componentes básicos y objetivos productivos deberían entenderse en estrecha dependencia con otros componentes si se quiere alcanzar su éxito y sostenibilidad (Peñuela, et al., 2014). Las transformaciones derivadas de la ocupación humana en la región tienden a restringirse al uso pecuario extensivo de los colonos antiguos en los hatos ganaderos, lo que se combina con la horticultura de conuco (pequeña parcela de tierra), o con la extracción o recolección, actividades más propias de los pueblos indígenas, pero que también se han adaptado a las nuevas condiciones de la cultura de los colonos en el territorio (Baptiste \& Ariza, 2008; Baptiste, 2015).

La sabana inundable está ligada a la cultura y la ganadería, constituye un depósito de actitudes, de conocimiento, de comprensión de la región y de valoración y disfrute escénico de los paisajes, con los contrastes propios del invierno y el verano. La cultura llanera resulta de un cúmulo de experiencias y vivencias de gentes que han convivido con el ecosistema durante siglos, encontrando formas de apropiarse del territorio y de vivir en función de su comprensión. La ignorancia en torno a esta cultura llanera y su menosprecio pueden repercutir en la destrucción de muchas comunidades y constituir un obstáculo para mejorar la ganadería (Ocampo \& Peñuela, 2014).

Retomando la propuesta antropológica más clásica, la etnografía recurre a un procedimiento metodológico orientado a comprender los "imponderables de la vida real", esto es, el sentido de la acción y lo que los sujetos realmente hacen en su vida cotidiana. Se fundamenta, como toda metodología cualitativa, en dos paradigmas científicos: el constructivismo y el 'interpretacionismo'. En los estudios etnográficos los protocolos de investigación suelen ser flexibles, pues no hay planteamientos predefinidos y se espera hasta el momento de contar con los hallazgos del trabajo de 
campo. A partir del marco teórico, se establecen "horizontes teóricos" para conocer la complejidad de los fenómenos que son objeto del análisis. Una vez realizado el trabajo de campo, los hallazgos se interpretan teniendo en cuenta sus múltiples relaciones e interpretaciones (Álvarez, 2011; Hammer \& Atkinson, 1994).

La subjetividad es la forma de constitución de una realidad social en sus diferentes aspectos: sociales, culturales y políticos, e implica, a la vez, una manera de pensar, sentir, comunicar y dar sentido e intencionalidad a las formas de interactuar y convivir del sujeto en la cotidianidad en la cual se gestan los procesos de interacción cultural. Es así que la subjetividad se expresa en las vivencias propias de cada individuo, como también en las construcciones colectivas generadas en la relación con el otro y lo otro (el contexto) (Álvarez, 2016)

En el presente estudio se plantea como problemática de partida el desconocimiento de la cultura y las costumbres de una población rural que ha permanecido aislada en el tiempo y el espacio, muchas de cuyas impresiones y valoraciones se desconocen, panorama que resulta difícil de aceptar desde la perspectiva de la investigación convencional. Se postula que es improbable alcanzar resultados productivos y técnicos si previamente no se tienen de presente aspectos de la cultura llanera araucana y su entorno ecosistémico. Por ello, el objetivo del estudio fue describir la etnografía del llanero habitante de los municipios de Arauca, Cravo Norte y Puerto Rondón, localizados en las sabanas inundables de Arauca, Colombia, a partir de los planteamientos de la tesis de maestría de Rodríguez (2018)

\section{Materiales y métodos}

Área de estudio. El estudio se desarrolló en los municipios de Arauca, Cravo Norte y Puerto Rondón en el departamento de Arauca, Colombia (Gobernación de Arauca, 2012). Inicialmente se proce-dió a elaborar un marco teórico que permitiera tener referentes útiles para analizar e interpretar la información suministrada, y establecer la finalidad del análisis de sis-temas y los conceptos de ecosistema, sistema región, sistema finca y agroecosistema. Asimismo, se hizo una revisión bibliográfica para documentar y describir el eco-sistema de sabana inundable, el sistema región y los seis agroecosistemas presentes en la sabana inundable: de caza, pesca y recolección, propios del indígena, conuqueros y de troja, mestizos (cruces del indígena y el blanco), y los pecuarios (porcinos, equinos y bovinos). Por último, se fue acopiando el material etnográfico mediante visitas a los municipios y entrevistas con los habitantes en esos ámbitos. Para sistematizar la información previa, se jerarquizaron los siguientes ejes temáticos: sabanas y cultivos, tipología productiva, pie de cría, manejos del rebaño, nutricional y sanitario, comercialización, uso de mano de obra, abigeato, presencia institucional, infraestructura vial y equipos, y cambio climático y arroz. Una vez transcrita la información recopilada, se procuró hilvanar las interpretaciones del investigador con las percepciones y puntos de vista de los entrevistados. Los fragmentos de sus intervenciones se resaltan con letra cursiva en el texto del manuscrito; sin embargo, dado que el análisis etnográfico sugiere el diálogo y la posibilidad de múltiples interpretaciones, en el anexo los interesados podrán encontrar el conjunto de las entrevistas y algún material fílmico.

Selección de las unidades de análisis y obtención de la información. Las personas entrevistadas se seleccionaron, en su mayoría, de la lista de ganaderos integrantes del Proyecto Bovino Arauca (PBA, 2015), aunque no se contempló exclusivamente esa condición. Se procuró hacer una selección según la disponibilidad de los productores y apelando a un conocimiento previo de sus nociones de la región. Se seleccionaron 30 productores, diez por cada municipio, y se los invitó a "un diálogo de saberes", en el ánimo de construir conocimiento con la participación de las comunidades y el aporte de sus vivencias y experiencias en torno a aspectos inherentes a su realidad cotidiana y a sus percepciones de lo que viven en el llano. A cada productor se le hizo una entrevista semiestructurada con final abierto (Hammer \& Wildavsky, 1990; Álvarez, 2011), que contenía 11 temas, para escuchar de viva voz sus propuestas concretas, surgidas del sentir "llanero anfibio", sobre los 11 ejes temáticos seleccionados.

La información se recolectó con la ayuda de video grabadora, cámara fotográfica y libreta de apuntes, describiendo y explicando detalladamente todos los posibles pormenores y fenómenos que arrojaran luz sobre las problemáticas del llanero. Los entrevistadores emplearon un lenguaje sencillo que respetaba la semántica llanera, buscando interesar al entrevistado. Los entrevistados se clasificaron según el tamaño del predio y el inventario ganadero en grandes productores (más de 1.000 bovinos), medianos productores (500 a 1.000 bovinos) y pequeños productores (menos de 500 bovinos) (Fedegán, 2014, 2016).

A cada ganadero se le asignó un número de entrevistado, con el nombre y el área del predio, la vereda, y el censo ganadero expresado en número de cabezas de ganado, y otras variables. A partir de esta información, y buscando dar cuenta de la subjetividad y la intersubjetividad del llanero de las sabanas inundables de Arauca, se interpretaron los datos y las observaciones provenientes de las entrevistas, y con ello se hizo la construcción de los resultados del presente estudio etnográfico del llanero araucano. Ante la necesidad de fundamentar el concepto del "otro sujeto", superando la oposición de los términos objetivo y subjetivo, se hizo preciso el empleo de la intersubjetividad, de manera que el estudio cumpliera con la condición de ser un producto social inseparable de la cultura llanera.

\section{Resultados y discusión}

Los resultados obtenidos a partir de los diálogos y encuentros con los 30 productores entrevistados, los cuales se registraron, como ya se mencionó, con videograbadora, 
material fotográfico y libreta de apuntes, arrojaron luces sobre el origen de los hatos y fundos araucanos. Se encontró que el $70 \%$ eran herederos directos de sus predios, y muchos de ellos, fundadores. Los antecesores, ya fallecidos, habían sido, en su mayoría, "fundadores de hato" que encontraron tierras sin títulos, sin habilitación y sin ganados, $\mathrm{o}$, en su defecto, herederos de personas que transmitieron sucesivamente bienes, ganados marcados y "derechos" a sus hijos y nietos, hasta alcanzar recientemente la legitimidad jurídica. Hasta hace muy poco, se legaba y "transfería" de palabra. La cultura y las costumbres de la tierra también eran derechos que se transmitían en el intento de ser llanero. Esto denota la reciente y aun incompleta conformación regional, resultado del mestizaje logrado de generación en generación, que, en ocasiones, alcanza un tercer o un cuarto grado. La evocación frecuente de "cuando los ancestros se fundaron", es algo recurrente en la memoria regional, lo que permite calcular la edad del llanero araucano, con cerca de 230 años de convivencia con el territorio.

Desde esos orígenes, la bicentenaria cultura llanera convive con un ecosistema natural, el cual sus exponentes han intentado domar paulatinamente o al que se adaptan y someten en la medida en que la naturaleza lo permite, cediendo hasta contar con las condiciones mínimas necesarias para la subsistencia y la seguridad alimentaria; en ocasiones, intentan aprovechar el excedente de alguno de los agroecosistemas que logran posicionar, como sucedió en el siglo antepasado con la ganadería bovina y la ternera a la llanera (o mamona llanera), la cual se vio realzada por la impronta de la gesta patriótica que también reclamó del hombre sabanero lo que el entorno exigía: el caballo y el ser llanero. La alianza entre el hombre y el caballo permitió dominar a las reses y convertirlas en el pilar fundamental de su economía.

Las entrevistas evidenciaron aspectos muy particulares, por ejemplo, la persistencia de una ganadería extensiva tradicional que, a diferencia de lo que sucede en otras regiones, no es provista o renovada a partir de las fincas y hatos: los predios no son ocupados y desocupados, como ocurre siempre. Por el contrario, los hatos son heredados con la tierra, van en las sucesiones, pasando de generación en generación, y por ello permanecen intactos muchos aspectos de las especies involucradas, conservando sus particularidades ancestrales, lo que las hace únicas. Una verdadera cultura del ganado que se hereda con todo lo que hay dentro.

El escaso conocimiento que se tiene sobre la producción pecuaria en la sabana inundable, principalmente en la academia, conduce a juicios exageradamente críticos y sesgados, pues sin conocerla, ni siquiera someramente, se califica a buena parte de los llaneros de latifundistas, hecho que no corresponde a la realidad, al ignorar que solo 30 de cada 100 hectáreas de terreno pertenecen a la unidad fisiográfica de bancos y banquetas, o áreas bien drenadas, correspondientes al terreno apto para el establecimiento de cultivos, como no sean los de arroz intensivo que ocupan toda el área, con las características típicas del monocultivo: destructivo, extractivo y voraz con la sabana. Esto no significa que este $30 \%$ de área "banqueada" se emplee en su totalidad para los cultivos, pues el conuco sabanero, que es el agroecosistema de cultivo sabanero por excelencia, ocupa apenas un pequeño porcentaje de ese banco, y su finalidad es la subsistencia. Al acopiar toda la información suministrada, se encontró que los 30 productores reunían un área total de unas 66.899 hectáreas, cuya legalización no ha sido del todo formalizada pese a haber tenido dominio sobre ellas durante más de cien años en algunos casos, lo cual confirma lo dicho sobre el manejo tradicional y la cultura llanera. En general, podría decirse que apenas 20.000 de las cerca de 67.000 hectáreas consideradas eran bancos, es decir, cerca del $30 \%$, tal y como lo afirmaron los productores. Guardando las mismas proporciones, se diría que en las 1.500.000 hectáreas que conforman la sabana inundable del departamento de Arauca, hay solamente 500.000 hectáreas en bancos, aproximadamente. "El resto es puro bajo", se oye repetir a los productores describiendo las características fisiográficas de sus predios.

En cuanto al inventario ganadero de los 30 entrevistados, que alcanzaba un total de 20.717 cabezas, si estas se distribuyeran en las 67.000 hectáreas, se encontraría una carga animal promedio de 0,3 cabezas por hectárea o, a la inversa, de 3,22 hectáreas por cabeza, lo que en el sistema convencional de producción bovina resulta extremadamente bajo e ineficiente. Por supuesto, dicha aseveración ignora que en la sabana nativa interactúan, por lo menos, seis agroecosistemas completamente interrelacionados e interdependientes en sistemas de pastoreo extensivo tradicional, además de la rica y abundante fauna nativa, que también ocupa el mismo espacio territorial.

La carga animal es una medida inconveniente para evaluar la productividad en la sabana inundable, pues no tiene en cuenta consideraciones muy particulares más valiosas que el criterio exclusivo de la producción bovina. En este sentido, uno de los entrevistados hizo el siguiente comentario: "Las cargas animales cambiaron. Ahorita queremos meterle a la sabana 1,5 y hasta 2 UGG por hectárea, y quién sabe cuántos animales por hectárea, y realmente la sabana produce, pero no se puede en cualquier pastura. Lo que hay que manejar en las pasturas son las cargas. Uno no puede apretar en carga, y menos la sabana porque ella es frágil. La sabana no se puede estrechar tanto con animales y con carga. La sabana hay que trabajarla al flujo que ella misma va dando" (Rodríguez, 2018). Esta observación hace referencia a la complejidad y la transversalidad del ecosistema anfibio llanero. Por lo tanto, el manejo en estos casos debe ir acompañado de una gran dosis de prudencia y comprensión, sobre todo de la cultura llanera ancestral, que, muchas veces, es la mejor opción, a pesar del bajo nivel tecnológico de la producción tradicional, el cual se ha clasificado como bajo y muy bajo para los hatos y fundos 
caracterizados por ser extensos y distantes de las cabeceras municipales y de las principales vías de acceso, lo que imposibilita la introducción de la tecnología, los equipos y la infraestructura necesarias para transformar la producción, y los animales de razas mejoradas o especializadas. Por ello, se ha optado por lo nativo, elección que ayuda a esclarecer el porqué de la vigencia de estos sistemas tradicionales que, no obstante, ya se enfrentan a la modernización y al riesgo de extinguirse.

Los niveles tecnológicos medio, alto, muy alto y óptimo se registran en situaciones particulares en las que las fincas y fundos tienen una cercanía relativa a los centros urbanos o a las vías principales $\mathrm{y}$, por ende, acceso a mejores posibilidades tecnológicas, instalaciones y equipos, y al manejo de herramientas más sofisticadas, con lo cual las prácticas tradicionales desaparecen o son reemplazadas, al igual que las especies y razas autóctonas, sustituidas por las especializadas modernas y más eficientes. En cuanto a los ingresos económicos y otras entradas adicionales de los productores, el análisis comprende desde el hato primitivo como único sustento, desde sus albores hasta hoy, cuando se impone paulatinamente la tercerización de la economía. En este sentido, se pudo establecer que solo cinco productores dependían de la ganadería como única fuente de ingresos: tres de Cravo Norte, uno de Arauca y uno de Rondón, en tanto que las actividades complementarias de comercio y empleo fueron reportadas por 24 de los 30 productores, lo que significa que su presencia en el predio es temporal y el desplazamiento a los sitios de trabajo es una constante determinada por la necesidad de conseguir ingresos adicionales. La actividad política es también complementaria $\mathrm{y}$, de hecho, se enmarca dentro de la economía terciaria. Dos de los entrevistados han incursionado en la política y por esa razón merecerían un análisis más detallado.

Por último, actividades como el arriendo parcial del predio para el cultivo del arroz son una realidad que puede ir en aumento en la medida en que se deterioren las condiciones económicas de los ganaderos, lo que debería ser objeto de estudio para buscar los mecanismos que consigan mantenerlos en su trabajo pecuario sin la presión de tener que arrendar su tierra a cualquier precio para un cultivo tan agresivo como el del arroz industrial, que sucede por la falta de oportunidades para el productor. Según el ingreso percibido por los productores, se analizaron las obligaciones que debe atender, entre ellas, la manutención y el sustento de casas y familias. En este sentido, la filosofía del hato se resumiría en la máxima de que cuanto más se gana más se gasta, ya que 11 fundos atendían las demandas de un pie de cría cercano a las 10.000 reses, en tanto que los nueve pequeños productores de Cravo Norte y Puerto Rondón, de economía familiar, solo atendían su predio. Entre estos dos extremos se sitúan los demás productores, que respondían por dos o tres casas, incluidas las casas de habitación urbana.

En cuanto a la tipología productiva, se encontró que las actividades más frecuentes eran la cría, el levante y el ordeño ya fuera para la elaboración de queso o para la leche; 16 de los 30 productores hacían el ordeño de manera artesanal para la producción de queso o la venta ocasional de leche, cuya comercialización se hacía también de forma artesanal, enfrentando a diario la competencia multinacional y los bajos precios característicos de la producción industrial. A pesar de tanta adversidad, los productores podrían acomodarse al mercado que prefiere el consumo de estos productos naturales. Se concluyó, asimismo, que las razas especializadas han estado desplazando a la nativa, lo que obliga al productor a ser más eficiente y a dedicarse a otras actividades además de la cría y el levante. La ceba en la sabana es una actividad complementaria que paulatinamente se ha ido posicionando, como en el caso de Rondón, en donde los productores se han visto obligados a utilizar pastos introducidos, a mecanizar la explotación del suelo, a utilizar sistemas de cerca eléctrica y rotación, elementos que en Cravo Norte no se conocen y en Arauca están ya comenzando a masificarse para contrarrestar la distancia del gran mercado, es decir, Bogotá y las grandes ciudades, transformaciones introducidas por la presión que ejerce Tame, el municipio del piedemonte más cercano a Puerto Rondón. A medida en que uno se adentra en la sabana, se hace menos frecuente el uso de estas ayudas tecnológicas y es menor el desarrollo económico. Asimismo, como parte de la visión sistémica propuesta para el análisis de la sabana inundable, se constató el uso de especies introducidas, cuyo comportamiento se mencionó en párrafos anteriores para explicar las razones de la desaparición de los recursos genéticos autóctonos y el predominio del ganado cebú que se ha extendido progresivamente en la sabana.

En cuanto a la venta de ganado en pie, se suele llevar a cabo la venta "al ojo", pues 17 de los 30 productores entrevistados dijeron que hasta hacía muy poco así vendían sus animales. Esto implica la renuncia al cálculo matemático de pesos y medidas, muchas veces por no contar con equipo adecuado como romanas y básculas, relativamente fáciles de conseguir en el mercado actual. El total de los productores de Cravo Norte vendían sus animales al ojo, mientras que en Arauca, lo habían hecho cinco de los 11 entrevistados, y en Puerto Rondón solo uno. Sin lugar a dudas, esto significa un gran atraso tecnológico y una muy limitada comercialización, pero, además, la existencia una cultura arraigada a las costumbres en donde campea la informalidad y el desconocimiento de los recursos con que se cuenta en los predios, estimulada también por la falta de precios favorables, lo que no estimula el mejoramiento ni una mayor eficiencia de la producción.

La distancia del predio al poblado más cercano, última variable de análisis, permite referirse al nivel de desarrollo de los fundos, hatos o fincas, entendiéndose este como la evolución progresiva de la economía hacia mejores niveles de vida. En este contexto, la relación entre el predio y la distancia es inversamente proporcional: a mayor distancia, menor nivel de desarrollo del predio. En este mismo sentido debe considerarse el comportamiento centrífugo del 
desarrollo, cuyo epicentro es la gran ciudad, es decir, Bogotá, con su mercado y su dinámica económica, que se desplazan organizadamente por el espacio territorial hacia el extremo oriental del país, atravesando Tunja y Sogamoso (Boyacá), luego Yopal y Corozal (Casanare), para cubrir después Tame y Puerto Rondón y seguir de forma paralela al río Casanare hacia el oriente (departamento de Arauca) y, en esa misma dirección, desde Saravena y Arauquita, al occidente del departamento, en sentido paralelo al río Arauca, hasta arribar finalmente a la capital Arauca y, por último, a Cravo Norte, al suroriente, y nuevamente hacia Casanare, aumentando la distancia y disminuyendo la dinámica económica y el desarrollo, el tamaño y la cantidad de las vías de acceso, lo que aleja el mercado, y las posibilidades que este brinda.

\section{Conclusiones}

La cultura llanera es una cultura primitiva que dificulta los procesos de mejoras en la producción. Cualquier intento por transformar la producción debe iniciarse con la implantación de registros y del control de pesos. El llanero anfibio ha tenido que cohabitar con el ecosistema de las sabanas inundables durante siglos, encontrando formas de apropiarse del territorio y de vivir en función de su comprensión.

El acceso de los productos de origen a los mercados podría ser una alternativa sostenible, ya que en las entrevistas se evidenció la capacidad que brinda la sabana de desarrollar el ciclo completo de producción bovina con un tipo de ganado, el "tameño", que está alcanzando reconocimiento, pero es preciso elaborar estrategias de comercialización para conquistar nuevos mercados para lo que puede ser un auténtico producto orgánico.

El potencial real de la sabana inundable está en el manejo complementario e integrado de todas estas especies y de la diversidad agroecosistémica, con lo que se lograría una mayor producción de biomasa y sería posible que cada especie aprovechara los diferentes nichos ecosistémicos, atendiendo así la seguridad alimentaria y fomentando la singularidad y la diversidad del ecosistema mediante iniciativas como el ecoturismo y la preservación de la cultura productiva generada en la relación del hombre con su medio. Probablemente el aprovechamiento de las especies equina, porcina y bovina, especialmente adaptadas al ecosistema, orgánicamente producidas, pueda generar "productos diferenciados y de origen", que, hasta el momento, no han recibido la atención debida en el marco de las estrategias de "fomento" del desarrollo regional.

\section{Contribución de los autores}

Luis Ernesto Rodríguez-Qüenza: participación en el análisis e interpretación de datos, en la redacción del artículo, en la recolección de resultados, y el aporte de material de estudio; Alonso Correa-Toro, Milena Hernández-Rodríguez: participación en la concepción y diseño del artículo, análisis e interpretación de datos; Arcesio Salamanca C.: revisión crítica del artículo, aprobación de la versión y corrección de estilo.

\section{Conflicto de intereses}

Los autores declaran no tener conflicto de intereses.

\section{Referencias}

Aguilera, J. (1989). Presente y futuro de la producción animal en el mundo con limitaciones y recursos alimenticios. Anales de la Academia de Ciencias veterinarias de Andalucía Oriental. 1: 52-63.

Álvarez, R. M. (2011). La investigación etnográfica: una propuesta metodológica para Trabajo Social. Revista Trabajo Social UNAM. 20: 73-97.

Álvarez, O.M.E. (2016). La subjetividad e intersubjetividad: un camino en la comprensión de lo cultural. Revista Linhas. 17 (34): 323-336.

Baptiste, B. (2015). Ambientes Radicales. Columna de Brigitte Baptiste, Instituto de Investigación de Recursos Biológicos Alexander von Humboldt. Fecha de consulta: marzo de 2019. Disponible en: http://www.humboldt.org.co/es/actualidad/ item/752-ambientes-radicales

Baptiste, B. \& Ariza, A. (2008). Ecología de las sabanas inundables de Casanare. $1^{\circ}$ Congreso de Sabanas Inundables, Universidad Cooperativa de Colombia, Facultad de MVZ Arauca.

Fedegán (2014). Estadísticas, Inventario ganadero. Fecha de consulta: agosto de 2018. Disponible en: http://www. fedegan.org.co/estadisticas/inventario-ganadero

Fedegán (2016). Estadísticas, Inventario ganadero. Fecha de consulta: agosto de 2018. Disponible en: http://www. fedegan.org.co/estadisticas/inventario-ganadero

Gobernación de Arauca. Plan de Desarrollo Departamental de Arauca 2012-2015-PBOT. (2012). Esquema de Ordenamiento Territorial Municipio de Cravo Norte. p. 12,13.

Hammer, D. \& Wildavsky, A. (1990). La entrevista semiestructurada de final abierto. Aproximación a una guía operativa. Historia y Fuente Oral. 4: 23-61.

Hammer, M. \& Atkinson, P. (1994). Etnografía; Métodos de Investigación. Ed. Paidós, Barcelona. p. 17-19.

Hart, D. R. (1985). Conceptos básicos sobre agroecosistemas. Centro Agronómico Tropical de Investigación y Enseñanza. Costa Rica, p. 1-10.

Jara, C. (2009). Reflexiones sobre la teoría de los campos mórficos y el desarrollo rural sostenible. Revista Desarrollo Territorial y Desarrollo Rural, CIDES UMSA, 2009

Johansen, O. (2004). Introducción a la Teoría General de Sistemas/ Introduction to The General Theory of Systems. México: Limusa. p. 53-66.

Machado, H. \& Campos, M. (2008). Reflexiones acerca de los ecosistemas agrícolas y la necesidad de su conservación. Revista Pastos y Forrajes. 31 (4): 307-320.

Malagón, R. \& Prager, M. (2001). El enfoque de sistemas: una opción para el análisis de las unidades de producción agrícola. Sede Palmira: Universidad Nacional de Colombia. p. 23-24.

Ospina, O. (2016). Diseño, implementación y evaluación del efecto de un sistema de gestión del conocimiento sobre las fuentes, nivel y uso del conocimiento en productores ovinocaprinos en Colombia. Bogotá: Universidad Nacional de Colombia, Facultad de Medicina Veterinaria y Zootecnia, Postgrado, Colombia. 
Proyecto Bovino Arauca - PBA. (2015). Desarrollo de un programa de gestión tecnológica para la innovación social y productiva de la carne y la leche en sistemas de producción bovina de la región de los llanos en Colombia. Departamento de Producción Animal, Facultad de Medicina Veterinaria y Zootecnia, Universidad Nacional de Colombia sede Bogotá.

Peñuela, L., Solano, C., Ardila, V., Galán, S. (2014). Sabana inundable y ganadería, opción productiva de conservación en la Orinoquia. Proyecto: "Fortalecimiento institucional y de política para incrementar la conservación de la biodiversidad en predios privados en Colombia”. Conservación de la biodiversidad en predios productivos. Serie $\mathrm{N}^{\circ}$ 3. 3: 230 .

Rodríguez, Q.L.E. (2018). Etnografía de la población rural del ecosistema de sabanas inundables en el departamento de Arauca. Tesis MSc. Universidad Nacional de Colombia. Bogotá. 